\title{
Virtual Reality as a Therapy Adjunct for Fear of Movement in Veterans With Chronic Pain: Single-Arm Feasibility Study
}

Christopher A Fowler ${ }^{1}$, PhD; Lisa M Ballistrea ${ }^{1}$, DPT; Kerry E Mazzone ${ }^{2}$, DPT; Aaron M Martin², PhD; Howard Kaplan $^{3}$, MEd; Kevin E Kip ${ }^{1,4}$, PhD; Katherine Ralston ${ }^{1}$, BSc; Jennifer L Murphy ${ }^{2,5}$, PhD; Sandra L Winkler ${ }^{1}$, OTR/L, $\mathrm{PhD}$

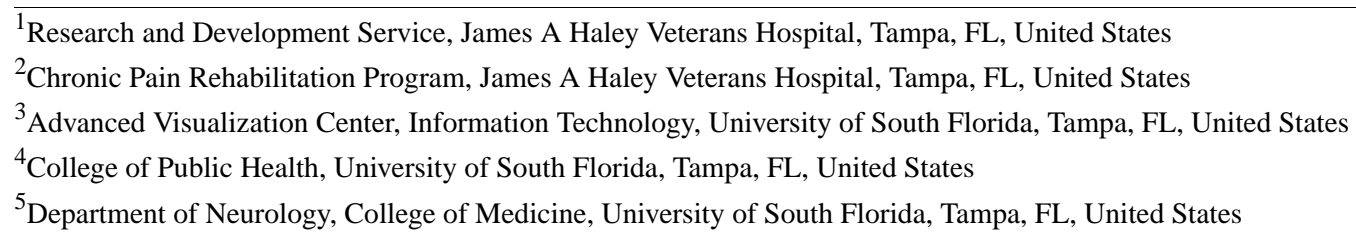

\section{Corresponding Author:}

Christopher A Fowler, $\mathrm{PhD}$

Research and Development Service

James A Haley Veterans Hospital

8900 Grand Oak Circle

Tampa, FL, 33705

United States

Phone: 18135583904

Email: christopher.fowler3@va.gov

\section{Abstract}

Background: Virtual reality (VR) has demonstrated efficacy for distraction from pain-related thoughts and exposure to feared movements. Little empirical VR research has focused on chronic pain management.

Objective: The purpose of this study was to examine the feasibility of VR as an adjunctive intervention for Veterans with chronic pain. We designed a hierarchy ranging from low-intensity pain distraction to high-intensity movement-based exposure for this purpose. VR apps were mapped onto the hierarchy.

Methods: Sixteen Veterans receiving inpatient chronic pain rehabilitation participated in daily VR sessions over a 3-week period. Trajectories across the distraction-to-exposure hierarchy and Veteran-reported intensity ratings were described and evaluated over time. Minimum clinically important differences (MCIDs), pre-post effect sizes, and 95\% confidence intervals were examined for fear of movement using the Fear of Daily Activities Questionnaire (FDAQ) and Pain Outcomes Questionnaire-VA (POQ-VA; fear scale). This approach was applied to secondary outcomes: POQ-VA (pain intensity, interference, negative affect), Pain Catastrophizing Scale, and Patient-Specific Functioning Scale (PSFS). Session attendance, completion, and VR experiences were described.

Results: Ten of 14 Veterans (71\%) who participated in three or more VR sessions completed the distraction-to-exposure hierarchy. Only three trajectories emerged more than once. Due to high completion rates, Veterans that completed the hierarchy could self-select nonhierarchy apps. Veterans rated all hierarchy levels (low, medium, high) near medium intensity. Self-selected activities were rated as high intensity. For kinesiophobia, six Veterans (38\%) exceeded the MCID on the FDAQ and a small effect size improvement was observed (Cohen $\mathrm{d}=-0.35$ ). The confidence interval (95\% CI -0.71 to 0.01 ) indicated the possibility of a null effect. The POQ-VA fear scale yielded no effect (Cohen $\mathrm{d}=0.06,95 \% \mathrm{CI}-0.43$ to 0.54 ). For secondary outcomes, Veterans exceeding MCID were calculated with complete data: pain intensity $(1 / 15,7 \%)$, pain catastrophizing $(5 / 14,36 \%)$, and patient-specific functioning $(10 / 15,67 \%)$. Effect sizes were large for patient-specific functioning $(\mathrm{Cohen} \mathrm{d}=1.14,95 \% \mathrm{CI}$ 0.50-1.78), medium for mobility interference (Cohen $\mathrm{d}=-0.56,95 \% \mathrm{CI}-0.96$ to -0.16 ), and small for pain intensity (Cohen $\mathrm{d}=-0.40,95 \% \mathrm{CI}-0.69$ to -0.12 ) and catastrophizing (Cohen $\mathrm{d}=-0.41,95 \% \mathrm{CI}-0.79$ to -0.02 ). No effects were observed for interference in daily activities (Cohen $\mathrm{d}=0.10,95 \% \mathrm{CI}-0.27$ to 0.47 ) and negative affect (Cohen $\mathrm{d}=0.07,95 \% \mathrm{CI}-0.26$ to 0.40 ). Veterans attended $85.2 \%$ (98/108) of VR sessions and completed 95\% (93/96) of sessions attended. Twenty-minute sessions were rated as too short. No significant adverse events were reported. 
Conclusions: Findings support the feasibility of VR as an adjunct for Veterans with chronic pain. However, the hierarchy will require modification, as evidenced by homogeneous intensity ratings. Veteran-selected activities presented the highest intensity ratings, largest outcome effect size (PSFS), and MCID. This highlights the important role of utilizing Veteran stakeholders in hierarchy modification, design of VR interventions, and outcome selection.

(JMIR Form Res 2019;3(4):e11266) doi: $10.2196 / 11266$

\section{KEYWORDS}

chronic pain; virtual reality; Veterans; pain management; rehabilitation; fear of movement; kinesiophobia; exposure therapy; distraction therapy

\section{Introduction}

\section{Virtual Immersion}

Virtual environments present an opportunity to safely and gradually expose Veterans with chronic pain to movements they avoid in the real world. Virtual reality (VR) describes "computer-generated simulations of three-dimensional objects or environments with seemingly real, direct, or physical user interaction" [1]. VR technologies use wearable devices to project a virtual environment and to track movements within it [2]. Sensory inputs (eg, visual, audio, tactile) give the user the illusion of "immersion" or being cognitively absorbed by a virtual environment [3,4]. Interaction with the immersive environment allows the user to co-create their experience giving them a subjective sense of "presence" in the virtual environment [3,5]. Immersion and presence can facilitate interventions using simulated 3D environments that set VR apps apart from in vivo behavioral treatments [6] and low-immersion 2D apps (eg, mobile phone) [7]. VR can serve as an adjunct to assist with the adoption of pain management skills in evidence-based interventions $[4,8]$.

\section{Virtual Reality for Pain Management}

Virtual reality apps have demonstrated efficacy and feasibility for delivering pain management skills, including distraction and exposure therapies. A rapid review (20 studies, $\mathrm{N}=337$ ) found short-term pain reduction (strong evidence) and analgesic effects (moderate evidence) [4]. A meta-analysis of controlled studies (16 studies, $\mathrm{N}=656$ ) estimated a medium effect size pain reduction when using VR during medical procedures [9]. To date, VR research has prominently focused on acute, not chronic, pain [4,9].

\section{Chronic Versus Acute Pain}

Pain is defined as "an unpleasant sensory and emotional experience associated with actual or potential tissue damage or described in terms of such damage" [10]. Postinjury acute pain is a nociceptive physiological warning to limit or avoid certain behaviors to prevent further harm [11]. Acute pain lasts 3 months or less and helps facilitate physical healing [12]. In chronic pain, generalized hypersensitivity in the central nervous system results in overactive pain [13] and sensory [14] pathways. Chronic pain persists beyond the physical healing process (ie, $>3$ months) and serves no adaptive purpose [12]. Still, chronic pain may feel indistinguishable from acute pain and leads people to avoid movement, which negatively impacts functioning $[13,15]$.

\section{Fear of Movement}

The fear-avoidance model of chronic pain posits a feedback loop with fear as a key component of avoidant behavior [16]. People with chronic pain mistakenly believe that pain sensations signal harm. Cognitive biases in pain processing (eg, behaviors that aggravate pain should be avoided to prevent reinjury) can result in kinesiophobia, or fear of movement, and subsequent pain avoidance as a means of self-protection $[16,17]$. Avoidance promotes a self-perpetuating cycle of physical deconditioning, negative affect, disability, and worse pain [17-19]. Interventions that operate on fear-avoidance principles (eg, graded physical therapy, cognitive behavioral therapy) aim to disrupt this cycle through gradual exposure to feared movements $[15,20]$. Reengagement in feared movements can modify pain interpretations (eg, pain signals harm), disrupt fear avoidance, and combat physical deconditioning [20-22]. This is often challenging because even safe movement can cause pain and emotional discomfort.

\section{Pain Distraction}

The most common VR intervention has been distraction therapy $[4,9]$ not exposure therapy. Distraction is hypothesized to be a mechanism of action for VR in attenuating pain [23]. Distraction therapy is based on the assumption that people have finite cognitive resources for information processing [24]. Immersive VR consumes cognitive and attentional resources through sensory input, thereby limiting pain-processing capabilities $[23,25]$. A meta-analysis ( 14 studies, $\mathrm{N}=581$ ) found large effect size reductions in acute and laboratory-induced pain when using VR distraction versus controls [26].

Distraction is important in the cognitive behavioral therapy for chronic pain protocol of Veterans Affairs (VA) [15]. A paucity of studies supports VR distraction for chronic pain. A within-group pilot study found a large effect size improvement in pain intensity following VR use [27]. A randomized crossover study found reduced pain intensity during VR compared with self-mediated distraction (eg, meditation, gaming), but not at posttest [28]. Still, VR provided superior distraction than self-mediated methods as evidenced by $56 \%$ less time thinking about pain [28]. Thus, VR distraction may be beneficial for chronic pain management.

\section{Graded Exposure}

People with chronic pain benefit from rehabilitation, not just immediate relief $[8,22]$. As such, passive pain distraction and more activating therapies, including exposure to feared movements, are used in comprehensive protocols [15]. Exposure therapy is important for chronic pain rehabilitation and 
compatible with VR [8,22]. A randomized controlled trial (RCT) assigned people with acute and chronic low back pain to receive 10 sessions of physical therapy with or without VR exposure ("virtual walking") [29]. Participants who received VR before each session experienced medium-to-large improvements in kinesiophobia, walking distance, and disability. A feasibility study of VR dodgeball found increased spine flexion, no adverse events, high acceptability, and likelihood to recommend the game to others with chronic back pain [30]. One study found that $76 \%$ of participants indicated a preference for VR exposure therapy over in vivo exposure before randomization [31]. Only $3 \%$ indicated that they would refuse VR compared with $27 \%$ for in vivo. This evidence supports VR exposure as an efficacious and acceptable adjunct for chronic pain management.

\section{Distraction-to-Exposure Hierarchy}

This study was informed by the fear-avoidance model of chronic pain [16], assuming that gradual exposure to feared movements using VR can improve kinesiophobia and pain outcomes. Still, people with chronic pain experience sensory exacerbation which may be a VR contraindication. Passive distraction therapy apps may be useful to gradually integrate Veterans to VR use. Thus, we created a hierarchy of increasing intensity based on sensory integration theory [32]. Veterans began with low movement-intensive VR distraction apps and could gradually progress to medium and high movement-intensity apps (exposure). This helps ensure that VR does not over- or understimulate the user.

\section{Gaps Addressed}

This study addresses notable gaps in VR research. First, there is a dearth of chronic pain studies in the growing literature about VR for pain management [9,33]. Second, VR studies have examined individual applications of pain distraction and exposure. Despite the efficacy of both therapies and distraction inherent in VR, no identified studies used a hierarchy approach [23]. Third, we failed to identify any VR pain management studies conducted with Veterans. More research is needed given the need for alternative nonpharmacological treatments for Veterans [34], who experience chronic pain with greater prevalence than non-Veterans [35].

\section{Specific Aims}

This study examined the feasibility of VR as an adjunct for chronic pain management. Evidence obtained is intended to inform a future RCT that will test the efficacy of VR and aim to validate our current and future revised distraction-to-exposure hierarchy. Primary aims are to (1) describe and compare the Veteran trajectories and self-reported app intensity ratings over a 3-week treatment period on the distraction-to-exposure hierarchy, (2) estimate the proportion of Veterans experiencing minimum clinically important differences (MCIDs) and within-subject effect size and $95 \%$ confidence intervals (CIs) for fear of movement and secondary pain outcomes associated with the use of VR, and (3) pilot test this protocol to assess the feasibility of VR use to plan for a future RCT.

\section{Methods}

\section{Study Setting}

This study was conducted in the Chronic Pain Rehabilitation Program at the James A Haley Veterans Hospital in Tampa, FL. This program used a cognitive behavioral approach to target the biopsychosocial impact of chronic pain [36]. Veterans were referred to this unique 19-day residential treatment program from throughout the VA system. For a more comprehensive overview of this program, see Murphy and colleagues [36,37].

\section{Participants and Recruitment}

Each week, up to four Veterans entered the 19-day Chronic Pain Rehabilitation Program, and up to four graduated and were discharged. All Veterans $(\mathrm{N}=18)$ that entered the program over the 3-week study period were targeted for recruitment; one Veteran refused. Of the 17 Veterans that consented, one did not meet the inclusion criteria. The final sample consisted of 16 Veterans (89\%). Inclusion criteria were (1) diagnosis of chronic pain syndrome (International Classification of Diseases [ICD], Ninth Revision, Clinical Modification code 338.4 and ICD-10 code G89.4) and (2) negative screen for illicit substances and unprescribed opioids. Exclusion criteria were uncontrolled medical and psychological factors (ie, aggression, depression, psychosis, suicidality) that could interfere with rehabilitation. These criteria mirrored that of the chronic pain program [36,37].

\section{Design}

A hybrid type 1 implementation-effectiveness design [38] was used to collect clinical efficacy and preimplementation data for VR. Qualitative and quantitative preimplementation data were collected following each VR session (aims 1 and 3). This paper emphasizes quantitative outcomes. Fear of movement and pain outcomes were assessed using a within-participants pretest-posttest design (aim 2).

\section{Intervention}

The distraction-to-exposure hierarchy was built with input from chronic pain program clinical stakeholders [39]. The hierarchy started with low stimulation intensity and then moved to high movement intensity. Twelve commercially available VR apps, six per head-mounted display (HMD), were then chosen to fit intensity levels. Low-intensity distraction apps included mindfulness meditation [40] and visual imagery $[41,42]$, which required minimal movement. Medium-intensity apps included virtual walking or swimming $[43,44]$ and controlling aircraft or watercraft $[45,46]$, which required head and neck movement. High-intensity apps were 3D painting $[47,48]$ and music or rhythmic-based $[49,50]$, which also required torso and upper extremity movement. Veterans alternated between two commercially available VR HMDs: Oculus Rift [51] and Samsung Oculus Gear VR [52]. Rift is an HMD with hand-tracking controllers, which is used with commercial gaming computers. Gear VR uses Samsung Galaxy Series mobile phones (S6 and above) to project virtual environments with sound. Both HMDs have been used for pain management research in medical settings [28,53,54]. Figure 1 shows our team members using each HMD. 
Figure 1. Samsung Oculus Gear VR with supplemental hand controller (left) and Oculus Rift (right).

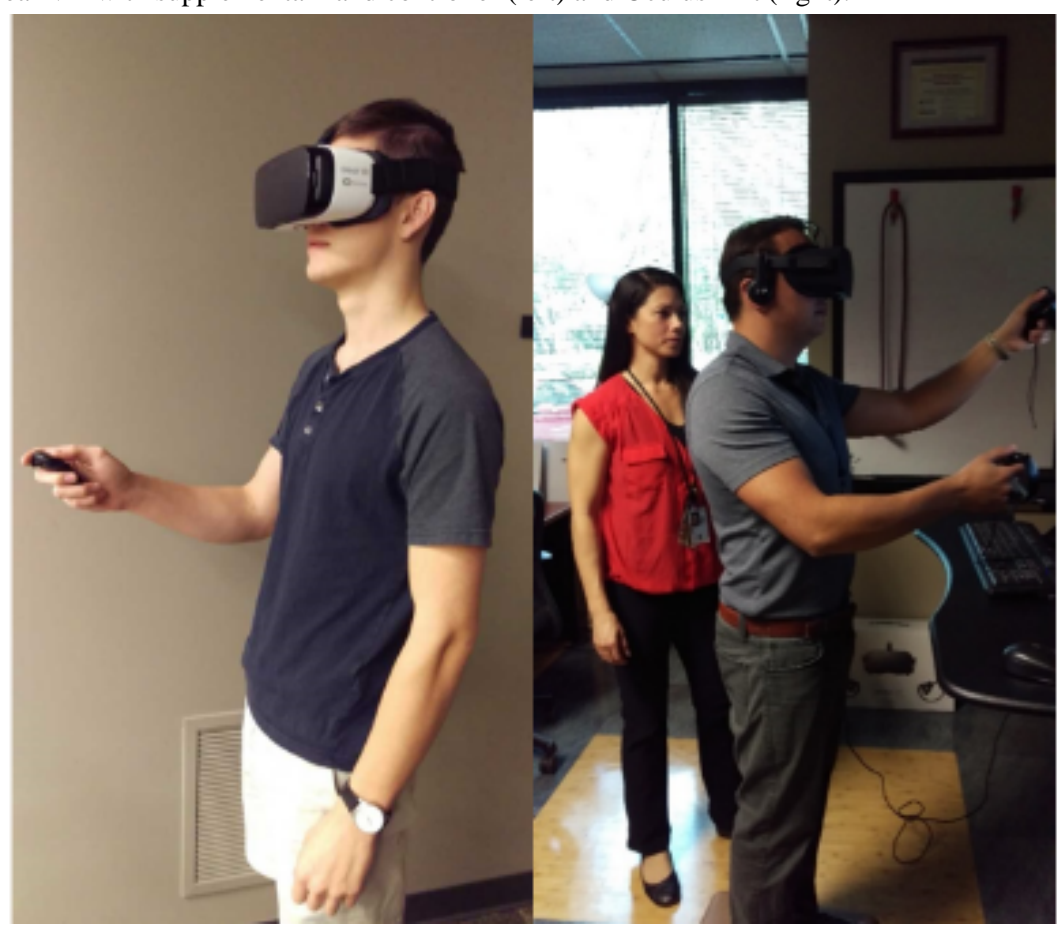

\section{Primary Measures}

\section{Daily Rating Form}

The eight-item daily rating form was created by the investigators to assess VR feasibility. Following each VR session, this form was used to track Veteran progress across the hierarchy (app selection), which HMD they used, and the number of sessions attempted and completed. Veterans reported their level of immersion using a single ordinal item adapted from Cole et al [55]: 1=aware and acknowledge the role of technology; $2=$ partially aware of technology, but perceived being inside a virtual environment; and 3=unaware of technology (complete virtual immersion). In addition, self-reported VR intensity (1=low, 2=medium, 3=high) and VR session length (1=too short, 2=just right, $3=$ too long) followed a similar approach [55]. Veterans could also provide feedback about their VR experience via three open-ended questions: likes, dislikes, and additional comments. This helped identify any facilitators (eg, HMD preferences), barriers (physical, psychological discomfort), and adverse events (eg, cybersickness, falls).

\section{Fear of Movement}

Kinesiophobia was assessed using two measures: the Pain Outcomes Questionnaire-VA (POQ-VA) [56] and Fear of Daily Activities Questionnaire (FDAQ) [57]. These scales had modest convergent validity $(r=.29, P=.28)$ suggesting they could be examined separately. Both scales have demonstrated acceptable psychometric properties in chronic pain studies [56,57].

The POQ-VA [56] is a multidimensional instrument developed specifically for the Veteran population. The fear subscale measures kinesiophobia using two items (fear of reinjury, safe to exercise) on Likert-type scales ranging from 0 to 10 and summed, with higher scores indicating positive outcomes. No MCID standards were identified.
The 10-item FDAQ [57] was designed in accordance with the fear-avoidance model. It was used to assess common feared movements (eg, sitting, standing, lifting, walking). All items are measured on scales anchored by 0 (no fear) and 100 (maximal fear) and then averaged. The MCID for the FDAQ is a 12.90-point reduction from baseline [57].

\section{Secondary Measures}

\section{Pain Outcomes Questionnaire-VA}

Secondary outcomes were examined to identify promising outcomes for use with a future VR RCT [39]. Multiple secondary pain outcomes were collected using the POQ-VA [56]. These were interference with activities of daily living and mobility as well as negative affect. No MCID scores were identified. The POQ-VA also assesses pain intensity using the common pain Numeric Rating Scale [58] ( $0=$ no pain at all to $10=$ worst possible pain). The Numeric Rating Scale MCID is 2.10 points for moderate pain (baseline $=4-6$ ) and 2.80 points for severe pain (baseline $\geq 7$ ) [59].

\section{Pain Catastrophizing Scale}

The 13-item Pain Catastrophizing Scale [60] was used to measure exaggerated beliefs about pain (eg, nothing I can do to reduce pain). Items are measured on a Likert-type scale anchored by 0 (not at all) and 4 (very) and summed with higher scores indicating maladaptive beliefs about pain. MCIDs of $38 \%$ or greater have been established for improved disability and pain intensity following pain rehabilitation [61].

\section{Patient-Specific Functional Scale}

The Patient-Specific Functional Scale (PSFS) [62] required Veterans to identify three activities that have been hindered because of their pain. These tasks were then rated 0 (unable to perform) to 10 (able to perform at prior level) and averaged. 
The MCID for the PSFS is 1.30 to 2.29 points (small), 2.30 to 2.69 (medium), and 2.70 or higher (large) [63].

\section{Procedures}

Veterans were informed about the study during orientation to the pain program. Consenting procedures were performed in-person by the research team before their first physical therapy session. All study procedures were approved by the James A Haley VA Research and Development Committee and the University of South Florida (Tampa) Institutional Review Board (protocol: 00031503).

Veterans completed 20 minutes of VR during daily physical therapy sessions. During session 1, the hierarchy was described to the Veterans, and they began with low intensity guided meditation. Each session, they were asked which intensity VR APPS they would like to use that session. Following each session, research staff administered the daily rating form. Primary and secondary outcome measures were administered to Veterans at intake and discharge (approximately 3 weeks) to the chronic pain program to track improvements. The research staff retrieved these data from the VA's electronic medical record.

\section{Statistical Analysis}

To address aim 1 (describe and compare the Veteran trajectories and self-reported app intensity ratings on the distraction-to-exposure hierarchy), distributions of the Veteran-selected apps (proposed intensity range 1-3) were plotted across VR sessions. The frequency of Veteran trajectories toward completing the hierarchy were counted to identify common patterns. Veterans that completed less than three VR sessions were excluded from this analysis because the hierarchy could not be completed in two sessions. Veterans' median self-reported app intensity ratings were also calculated and plotted across the first nine VR sessions. Sessions 10 and 11 were excluded due to the low frequency of Veterans $(\mathrm{N} \leq 2)$ that attended more than nine sessions. Consistencies across proposed and self-reported VR app intensity were descriptively compared.
For aim 2 (estimate the proportion of Veterans experiencing MCIDs and within-subject effect size and 95\% CI for fear of movement and secondary pain outcomes associated with the use of VR), changes in fear of movement and secondary outcomes were calculated and compared with established MCID scores for each respective measure excluding imputed missing values. The proportion of Veterans that exceeded MCID was calculated for each outcome. Within-participants Cohen $d$ effect sizes ([post mean-pre mean]/SD difference) and 95\% CI for fear of movement and secondary outcomes were calculated to examine the efficacy of VR [64,65]. Suspected outliers were assessed using multiple criteria. These included examining boxplots of these pre-to-post test change scores, Tukey fences [66], and clinical observation and consultation noted during the study. In the case of a suspected outlier, findings were reported with and without its inclusion.

For aim 3 (pilot test this protocol to assess the feasibility of VR use), compliance for the Veteran sample was calculated via the proportion of VR sessions attended (sessions attended/total scheduled sessions). Adherence was calculated using the proportion of completed 20-minute VR sessions (full sessions completed/sessions attended). Veteran ratings of the length of the session and their self-reported levels of immersion were plotted across sessions.

\section{Availability of Data and Materials}

The final deidentified datasets from this study (qualitative and quantitative) and the VR user manual will be made available by the corresponding author on reasonable request.

\section{Results}

\section{Demographic Characteristics}

Veterans ranged from 28 to 63 years, with a mean age of 49 (SD 12) years (Table 1 ). They were prominently male, and their racial/ethnic composition was primarily Caucasian or white. Median pain duration was 16.50 (IQR 14.62) years and baseline pain intensity was near the severe range $(\geq 7)$ on the Numeric Rating Scale [38]. The primary pain location was low back; daily opioid use was low. 
Table 1. Demographic characteristics for study sample $(\mathrm{N}=16)$.

\begin{tabular}{|c|c|}
\hline Characteristic & Participants \\
\hline Age (years), mean (SD) & $48.88(11.62)$ \\
\hline \multicolumn{2}{|l|}{ Gender, n (\%) } \\
\hline Female & $3(19)$ \\
\hline Male & $13(81)$ \\
\hline \multicolumn{2}{|l|}{ Ethnicity, n (\%) } \\
\hline African American or black & $4(25)$ \\
\hline Caucasian or white & $8(50)$ \\
\hline Hispanic or Latino & $2(12)$ \\
\hline Other & $2(13)$ \\
\hline \multicolumn{2}{|l|}{ Pain location, n (\%) } \\
\hline Head & $2(13)$ \\
\hline Low back & $11(69)$ \\
\hline Other & $3(19)$ \\
\hline Pain chronicity (years), median (IQR) & $16.50(14.62)$ \\
\hline Virtual reality sessions, median (IQR) & $7.50(6.50)$ \\
\hline
\end{tabular}

\section{Aim 1: Describe and Compare the Veteran Trajectories and Self-Reported App Intensity Ratings on the Distraction-to-Exposure Hierarchy}

In total, 10 of 14 Veterans (71\%) who participated in three or more VR sessions completed the hierarchy. Eleven different trajectories emerged among these 14 Veterans during completion of the hierarchy. Only three patterns (21\%) occurred more than once, which indicated notable variability. Six of the initial eight Veterans $(75 \%)$ reached the highest level of the hierarchy during the first week of testing. Because of this high frequency early in the study, Veterans that completed the hierarchy could self-select VR activities (eg, fishing, basketball free throws) in addition to hierarchy apps. Self-selected apps were assigned a fourth intensity level indicating they were beyond the hierarchy. Median progressions across the distraction-to-exposure hierarchy are presented in Figure 2.

Figure 2. Median Veteran progression across the distraction-to-exposure hierarchy. App movement intensity: 1=low, 2=medium, 3=high, 4=self-selected. VR: virtual reality.

\section{- Oculus Rift $\quad$ Gear VR $\square$ Aggregate}

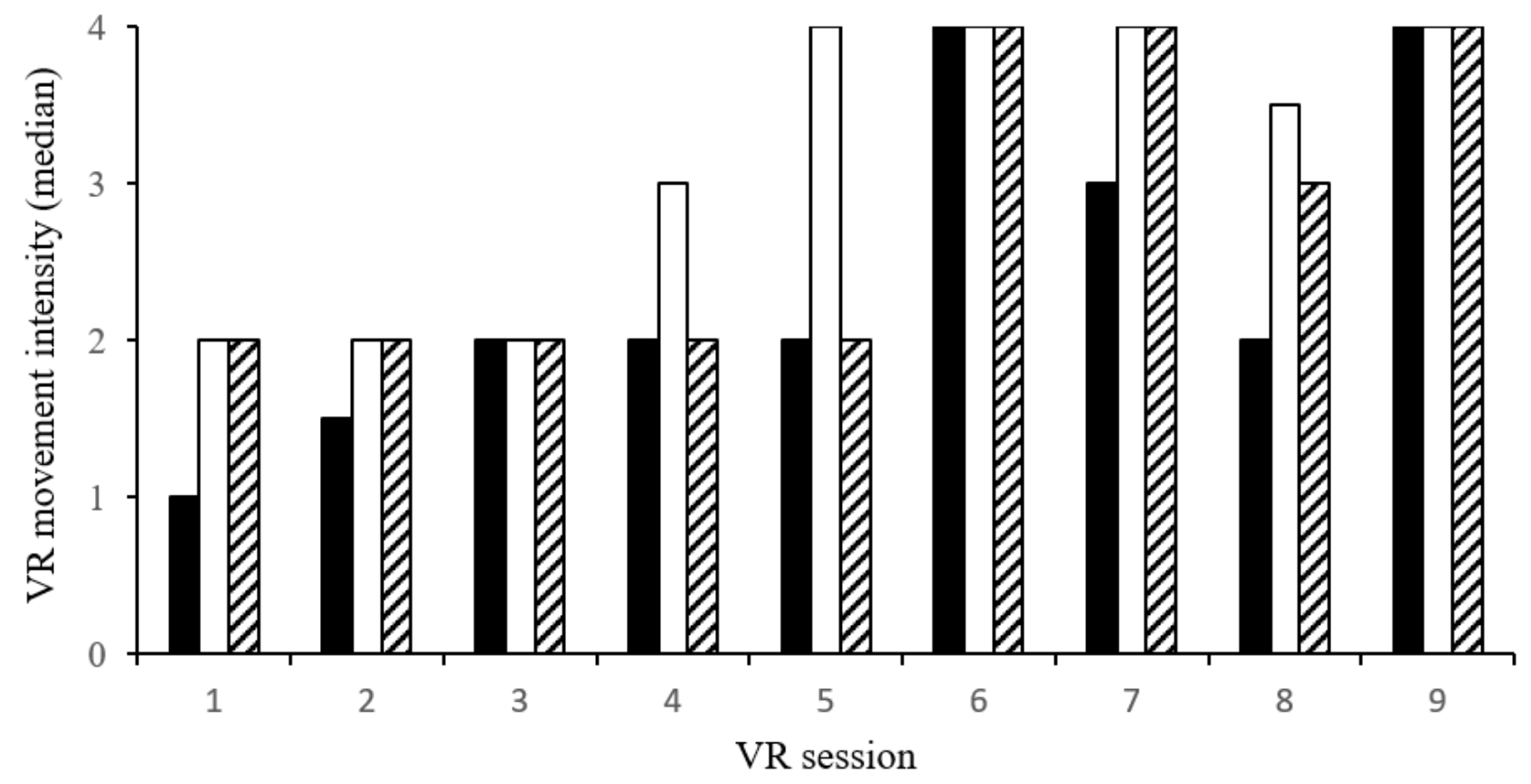


Veterans rated the intensity of each VR session. Low- to medium-intensity ratings were reported for each of the first four sessions (median 2, IQR 1.5). Sessions five to nine were rated from medium to high intensity (median 3, IQR 1; see Figure 3). Comparisons between Veterans' self-reported intensity ratings and hierarchy movement intensity levels were examined. Veterans rated low movement intensity apps (ie, distraction) between low and medium intensity (median 1.5, IQR 1). Both medium- (median 2, IQR 1) and high-intensity (median 2, IQR 1) movement exposure apps were rated as medium intensity. Veteran-selected apps were rated as high intensity (median 3, IQR 1). Veteran intensity ratings across hierarchy levels are presented in Figure 4.

Figure 3. Veteran-reported intensities for virtual reality (VR) apps across sessions. Self-reported app intensity: 1=low, 2=medium, 3=high.

- Oculus Rift aGear VR a Aggregate

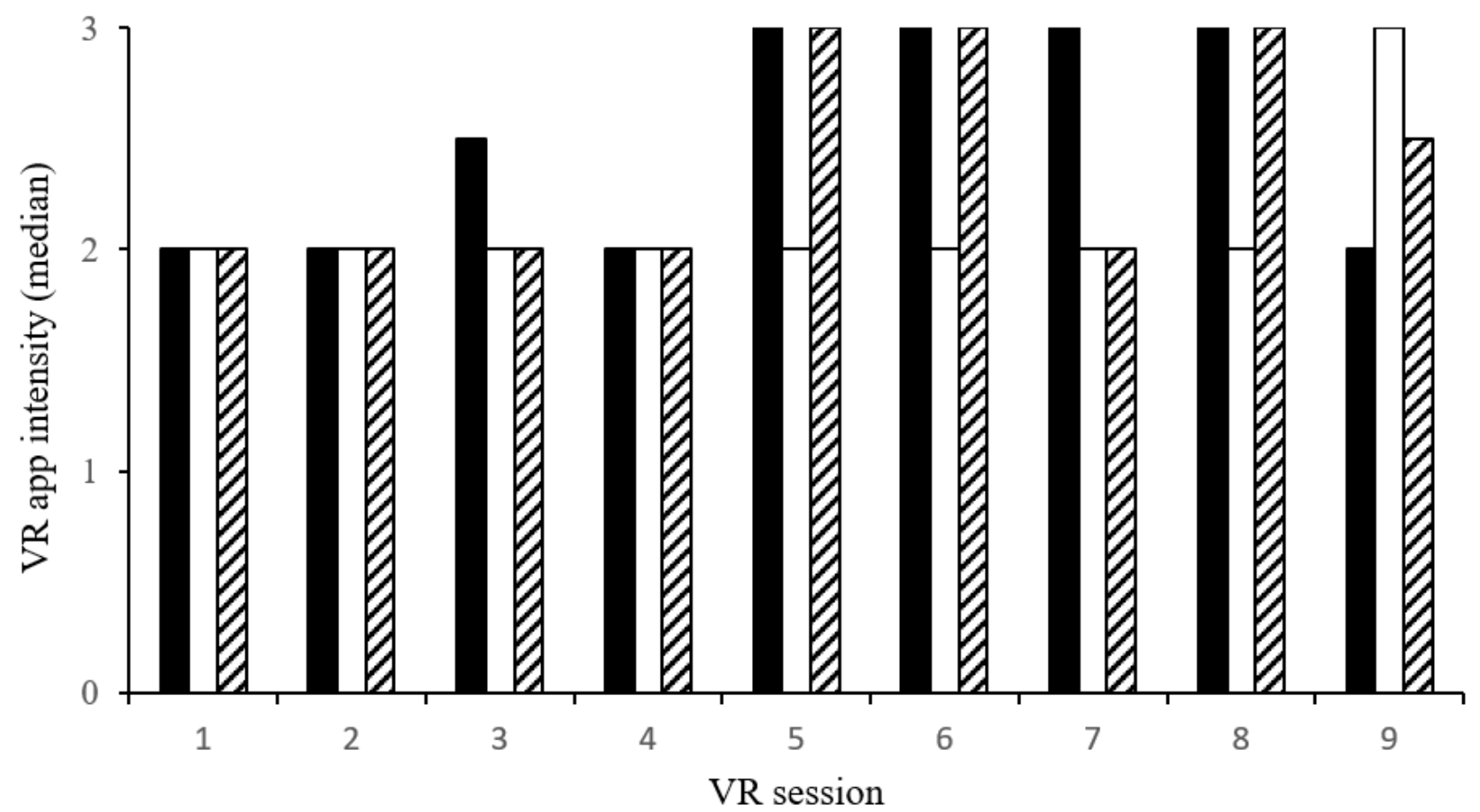

Figure 4. Veteran-reported intensities for virtual reality (VR) apps across movement intensity levels.

口Oculus Rift $\quad$ aGear VR a Aggregate

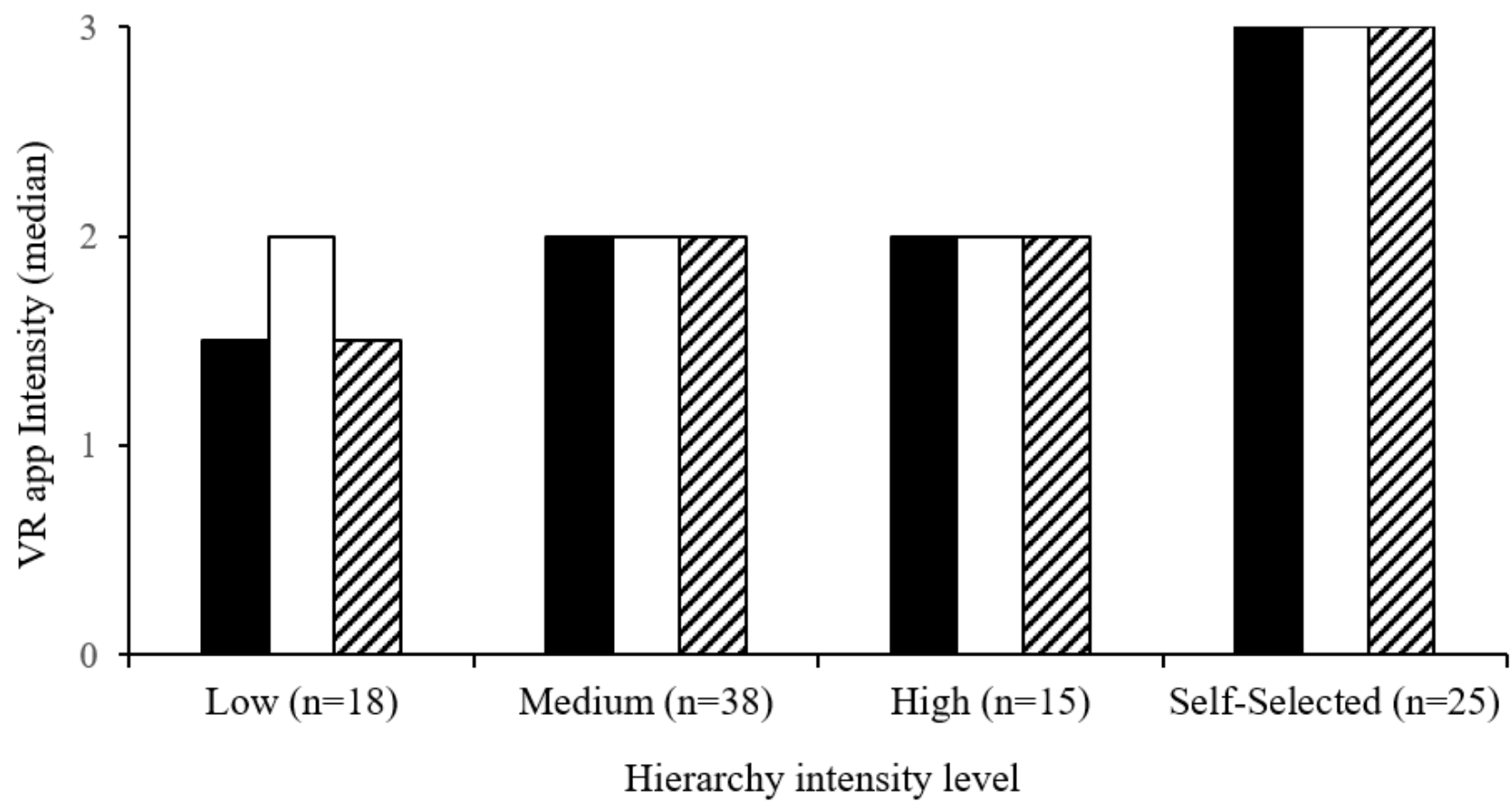




\section{Aim 2: Estimate the Effect Size, 95\% CI, and Proportion of Veterans Experiencing Minimum Clinically Important Difference for Fear of Movement and Pain Outcomes With Virtual Reality Use}

For the primary outcome kinesiophobia, 10 of 16 Veterans (63\%) had improved scores from baseline with six (38\%) exceeding an MCID of 12.9 points or greater [58]. The observed effect size improvement was minimal (ie, Cohen $d<0.20$ ) on the FDAQ (Cohen $d=-0.15,95 \% \mathrm{CI}-0.55$ to 0.25 ). One Veteran was identified as a possible outlier. When this score was excluded, a small effect size reduction in fear of movement was observed (Cohen $d=-0.35,95 \%$ CI -0.71 to 0.01 ). However, there was notable variability in the confidence interval with the effect size ranging from no effect to a medium improvement suggesting a possible null finding.

Using the POQ-VA fear scale, there was little evidence of a reduction in kinesiophobia (Cohen $d=-0.10,95 \%$ CI -0.69 to 0.48 ). When a possible outlier score was excluded, the effect size changed directions but did not amount to a notable effect (Cohen $d=0.06,95 \% \mathrm{CI}-0.43$ to 0.54 ). Full sample scale scores for primary and secondary measures, effect sizes, $95 \%$ CIs, and MCID are presented in Table 2.

Table 2. Baseline and posttest scores for fear of movement and secondary outcomes ( $\mathrm{N}=16)$.

\begin{tabular}{|c|c|c|c|c|}
\hline Measure & Baseline & Posttest & Effect size $(95 \% \mathrm{CI})$ & $\mathrm{MCID}^{\mathrm{a}}, \mathrm{n}(\%)$ \\
\hline \multicolumn{5}{|l|}{ Kinesiophobia, mean (SD) } \\
\hline FDAQ $^{b}$ & $59.20(24.83)$ & $56.45(21.02)$ & $-0.15(-0.55,0.25)$ & $6(38)$ \\
\hline POQ-VA ${ }^{\mathrm{c}}$ Fear & $12.44(5.01)$ & $11.91(3.01)$ & $-0.10(-0.69,0.48)$ & $\mathrm{N} / \mathrm{A}^{\mathrm{d}}$ \\
\hline \multicolumn{5}{|l|}{ Outliers excluded $^{\mathrm{e}}$} \\
\hline FDAQ & $60.28(25.31)$ & $54.75(20.58)$ & $-0.35(-0.71,0.01)$ & ${ }^{\mathrm{f}}$ \\
\hline POQ-VA Fear & $12.31(2.65)$ & $12.07(4.95)$ & $0.06(-0.47,0.59)$ & - \\
\hline \multicolumn{5}{|c|}{ Pain outcomes (POQ-VA), mean (SD) } \\
\hline Interfere daily living & $16.44(12.36)$ & $17.33(12.03)$ & $0.10(-0.27,0.47)$ & N/A \\
\hline Interfere mobility & $26.31(9.58)$ & $22.25(10.82)$ & $-0.56(-0.96,-0.16)$ & N/A \\
\hline Negative affect & $28.56(10.96)$ & $29.09(11.87)$ & $0.07(-0.26,0.40)$ & N/A \\
\hline \multicolumn{5}{|c|}{ Numeric Rating Scale, mean (SD) } \\
\hline Pain intensity & $6.88(1.26)$ & $6.38(1.59)$ & $-0.48(-0.87,-0.10)$ & $1(7)$ \\
\hline Outlier excluded & $6.73(1.16)$ & $6.40(1.64)$ & $-0.40(-0.69,-0.12)$ & - \\
\hline $\mathrm{PCS}^{\mathrm{g}}$ & $28.83(10.39)$ & $24.54(15.45)$ & $-0.41(-0.79,-0.02)$ & $5(36)$ \\
\hline PSFS $^{\text {h }}$ & $3.60(1.59)$ & $5.98(2.37)$ & $1.14(0.50,1.78)$ & $10(67)$ \\
\hline
\end{tabular}

${ }^{a}$ MCID: minimum clinically important difference.

${ }^{b}$ FDAQ: Fear of Daily Activities Questionnaire.

${ }^{\mathrm{c}}$ POQ-VA: Pain Outcomes Questionnaire-VA.

${ }^{\mathrm{d}}$ Not applicable.

${ }^{\mathrm{e}} \mathrm{N}=15$.

${ }_{\mathrm{f}} \mathrm{MCID}$ not recalculated.

${ }^{\mathrm{g}}$ PCS: Pain Catastrophizing Scale.

${ }^{\mathrm{h}}$ PSFS: Patient-Specific Functioning Scale.

\section{Aim 2: Secondary Outcomes}

The POQ-VA pain interference with mobility and activities of daily living as well as negative affect scales were examined. Veterans experienced a medium effect size improvement in interference with mobility (Cohen $d=-0.56,95 \% \mathrm{CI}-0.96$ to -0.16 ). There was variability with the effect size interval ranging from a small to large effect that was statistically significant $(P<.05)$ because the interval did not contain the null value of zero. Conversely, Veterans experienced a slight exacerbation in interference with activities of daily living (Cohen $d=0.10$, $95 \% \mathrm{CI}-0.27$ to 0.47 ). A similar pattern was observed with negative affect (Cohen $d=0.07,95 \% \mathrm{CI}-0.26$ to 0.40 ). Intervals for the latter two findings suggested no effect.

Five of 15 Veterans (33\%) with complete data had reduced pain intensity scores from baseline using the Numeric Rating Scale. However, only one Veteran (7\%) met MCID of 2.10 points or greater for moderate pain intensity at baseline (rating=4-6) [59] . No Veterans with severe baseline pain (rating 27 ) exceeded the MCID [59]. The sample had a small to medium improvement in pain intensity (Cohen $d=-0.49,95 \% \mathrm{CI}-0.87$ to -0.11 ). The confidence interval suggested a minimal-to-large effect. When a possible outlier was excluded, a similar effect pattern remained (Cohen $d=-0.40,95 \%$ CI -0.69 to -0.12 ). These effects were 
statistically significant $(P<.05)$ because the intervals did not contain the null value of zero.

Ten of 14 Veterans $(71 \%)$ had reduced catastrophizing scores from baseline, and five (36\%) exceeded the MCID of $38 \%$ or greater improvement [61]. Veterans experienced a small to medium effect size improvement in pain catastrophizing (Cohen $d=-0.41,95 \% \mathrm{CI}-0.79$ to -0.02 ). The interval ranged from a minimal to large and was statistically significant.

Finally, 14 of 15 Veterans (93\%) reported improvements in patient-specific functional tasks that were previously hindered by their pain, as measured by the PSFS. Ten Veterans (67\%) exceeded the MCID. Based on the scheme described by Abbott and Schmitt [63], Veterans MCID improvements were categorized as small (1.30-2.29; $n=2)$, medium (2.30-2.69; $n=2)$, and large $(\geq 2.70 ; n=6)$. The observed effect size improvement in patient-specific functioning ranged from medium to large and was statistically significant (Cohen $d=1.14$, 95\% CI $0.50-1.78, P<.001)$.

\section{Aim 3: Pilot Test the Protocol to Assess the Feasibility of VR Use}

Quantitative feasibility outcomes included levels of VR compliance, adherence, and session experiences. The compliance rate for this study $(85.2 \%$ ) was calculated via the number of VR sessions Veterans attended $(n=98)$ divided by the total number of scheduled sessions $(n=115)$. Of the 98 sessions attended, Veterans completed the full 20 minutes in 93 for an estimated adherence rate of $94.9 \%$. The most common reason for missing $(n=7)$ or shortened VR sessions $(n=2)$ was physical therapy-related (eg, longer than expected sessions). Compliance $(98 / 108,90.7 \%)$ and adherence $(93 / 96,96.9 \%)$ rates were calculated accounting for physical therapy as the primary reason for missing VR sessions.

The VR experiences included Veteran-rated immersion, session length, and HMD preferences, and were plotted across sessions. Adverse events were also described. During sessions 1 to 3, the Veteran's median immersion ratings (median 2, IRQ 1) indicated that they were aware of using technology and were immersed in the virtual world. Sessions 4 to 9 saw ratings vary to include greater immersion in the virtual world with less technology awareness (range 2-3). Overall, median immersion ratings for the Oculus Rift and VR Gear HMDs were equal (median 2, IQR $1)$. Veterans typically rated Oculus Rift higher across the first five sessions and Gear VR higher across sessions 6 to 9 (Figure 5). Median ratings indicated that 20-minute sessions typically were "too short" (median 1, IQR 1) with no sessions rated lower than "just right." Overall ratings for each HMD were equal (median 1, IQR 1), although median ratings for Gear VR were occasionally lower (ie, too short) than Oculus Rift across sessions (Figure 6).

No significant adverse events, such as falls, occurred. Minor adverse events included cybersickness symptoms (dizziness: $\mathrm{n}=2$ sessions; nausea: $\mathrm{n}=4$ sessions) and one session being terminated early because the Veteran was "starting to feel the weight [Gear VR] on their neck." Interestingly, 2 of 16 Veterans (13\%) purchased VR headsets during the study. Four additional Veterans (25\%) requested VR purchasing information and were provided with a handout (eg, models, cost, reviews) on request.

Figure 5. Veteran-rated immersion across virtual reality (VR) sessions. Immersion rating scale: $1=$ using technology, $2=$ using technology and immersed in the virtual world, $3=$ completely immersed in the virtual world.

- Oculus Rift $\square$ Gear VR घAggregate

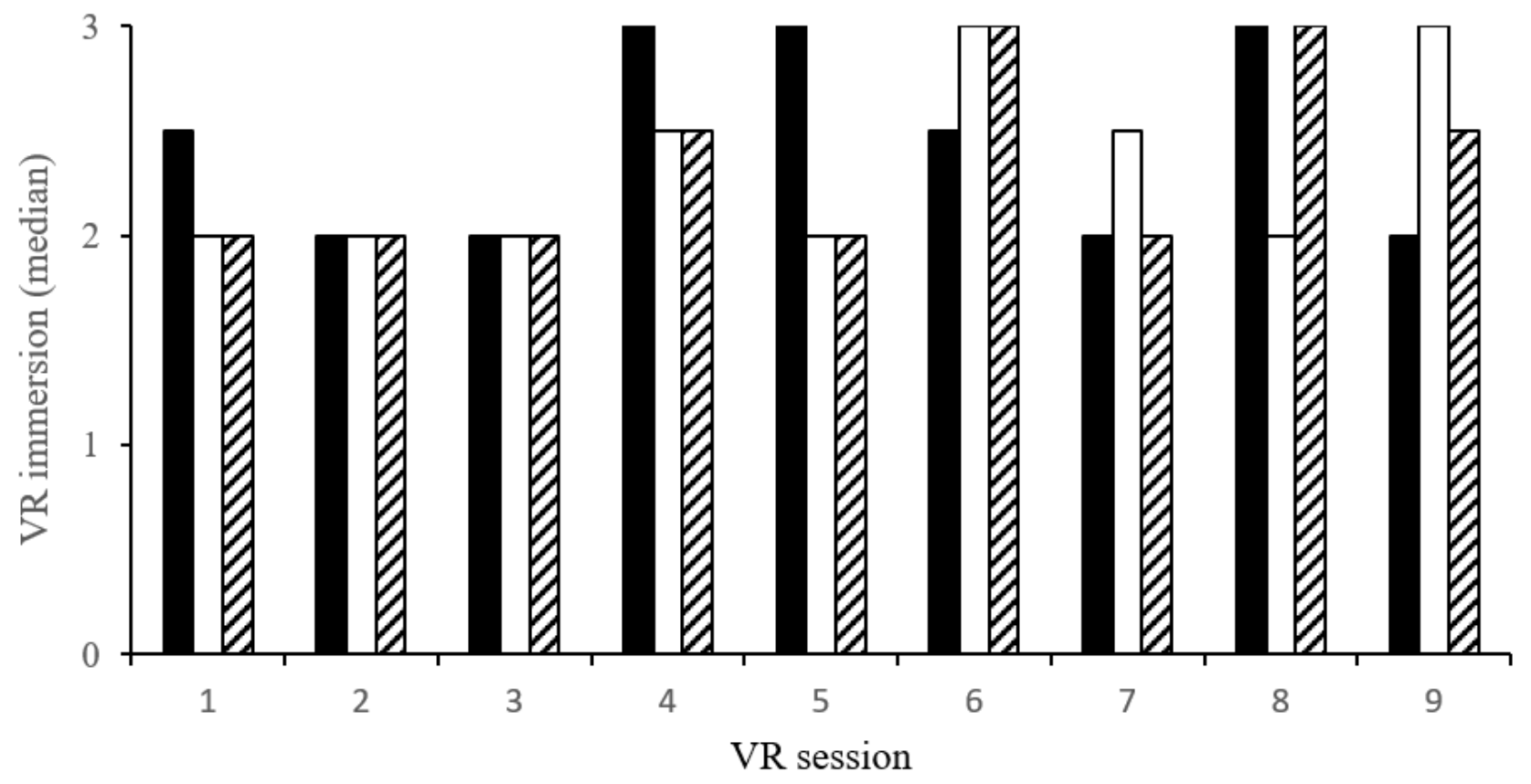


Figure 6. Veteran-rated virtual reality (VR) session length. Session length: $1=$ too short, $2=$ just right, $3=$ too long.

\section{-Oculus Rift $\square$ Gear VR $\square$ Aggregate}

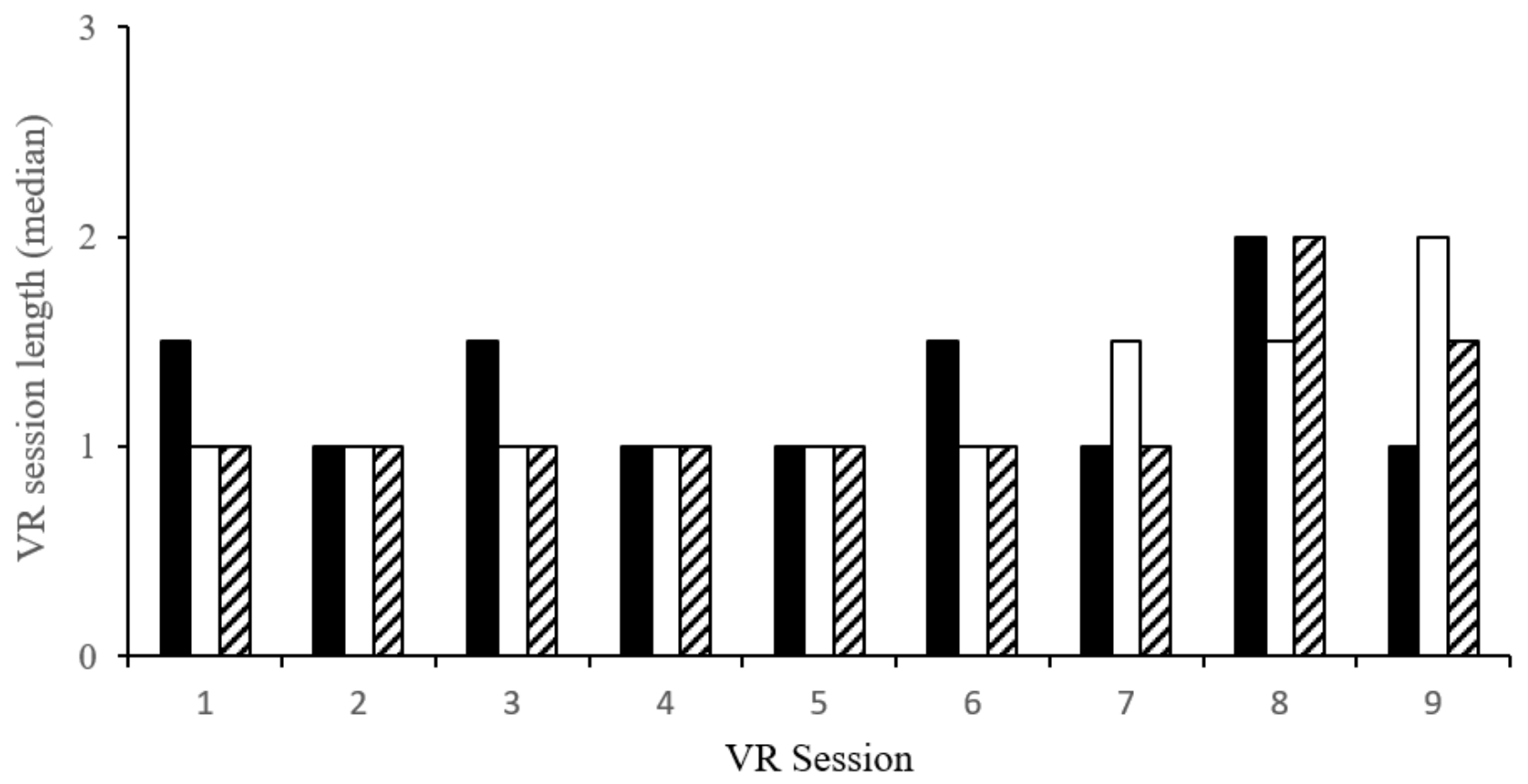

\section{Discussion}

\section{Principal Findings}

This study examined the feasibility of using a VR distraction-to-exposure hierarchy to improve fear of movement for Veterans with chronic pain. Descriptive findings suggest notable variability in Veteran trajectories across the hierarchy and that exposure apps requiring increased levels of movement were less challenging than anticipated. For the primary outcome (fear of movement), fear of daily activities, which measures specific movements, may be a more promising measure than the POQ-VA fear scale, which contains more general items (ie, exercise, reinjury). Promising pain outcomes for future research include interference with mobility, pain intensity, pain catastrophizing, and patient-specific functional activities. Veterans rated feeling immersed in VR even when they were cognizant of their technology use. They also rated their sessions as too short. Minimal adverse events were reported. This provided favorable evidence for the feasibility of VR for chronic pain management in a VA medical center. However, app selection, particularly for high movement intensity, will require modification before future research to align with the initial hierarchy.

\section{Trajectories and App Intensity}

The first aim of this study was to describe trajectories and self-reported app intensity ratings across the distraction-to-exposure hierarchy. Clinical consultation suggested that the majority of Veterans may not complete the hierarchy due to fear of movement. However, most Veterans reached or surpassed the highest movement level in six sessions. Differences between the Oculus Rift and Gear VR HMDs revealed most Veterans completed the hierarchy after only four sessions using Gear VR versus six sessions using the Oculus
Rift. To help ensure Veterans did not lose interest in the study if they found the tasks to be too easy, the hierarchy was expanded to include Veteran-selected activities (eg, fishing, basketball free throws) once they completed the hierarchy.

To support our hierarchy, we anticipated that median self-reported app intensities would align with movement intensity on the same rating scale (1=low, 2=medium, 3=high). Self-reported app intensity ratings suggest that hierarchy apps did not calibrate as expected. Low movement distraction apps were rated more intensely than expected, particularly for Gear VR. Medium movement-intensity apps were rated as expected. Proposed high movement-intensity apps were rated similarly to medium intensity, which indicated that this was not a unique intensity level as anticipated. Similar Veteran ratings across proposed levels of the hierarchy indicate a possible range restriction in movement intensity. Median high intensity scores were not observed until Veterans engaged in self-selected activities. Despite high intensity ratings, post hoc integration of these apps into the distraction-to-exposure hierarchy was difficult due to their heterogeneity.

\section{Fear of Movement and Pain Outcomes}

The second aim was to estimate the effect size, $95 \% \mathrm{CI}$, and proportion of Veterans experiencing MCID for fear of movement and pain outcomes. These analyses were to inform design and sample size requirements for a future trial and selection of appropriate instruments for use with VR for chronic pain. The MCID for kinesiophobia indicated that $38 \%$ exceeded MCID on the FDAQ. After removal of an outlier, a small effect size improvement was observed for the primary outcome fear of movement using the FDAQ, but not the POQ-VA. However, there was a wide confidence interval associated with these effect sizes (and pain outcomes), which is common in small sample sizes [67]. 
For secondary pain measures, the MCID statistics, effect size, and confidence interval estimates were generally promising. For pain outcomes, $36 \%$ of Veterans exceeded MCID in catastrophizing, but only one met MCID for pain intensity. In total, $67 \%$ of Veterans experienced MCID for patient-specific functional outcomes, thus indicating the most promising outcome measure in this small study.

On the POQ-VA, improvements were observed in interference with mobility (medium) and pain intensity (small). The $95 \%$ CIs were statistically significant for these outcomes. No changes were observed for negative affect or interference with activities of daily living. There was a small effect size improvement for pain catastrophizing and the significant $\mathrm{CI}$ ranged from minimal to large. Similar to MCID, the most promising improvements were for patient-specific functional outcomes. A large effect size was observed in patient-specific functional outcomes with the confidence interval ranging from medium to large.

\section{Feasibility of Virtual Reality}

The final aim was to establish the feasibility of VR as a therapy adjunct. All Veterans completed the study. Session attendance was over $85 \%$ and increased to more than $90 \%$ after accounting for sessions missed for physical therapy. Adherence, as measured by session completion, was nearly $95 \%$ and approximately $97 \%$ when accounting for therapy. This adjustment was made because VR was proposed to complement evidence-based interventions, and we did not want to study participation to reduce therapy adherence. Immersion ratings indicated that Veterans simultaneously felt immersed in the virtual world and that they were using VR HMDs. Median immersion ratings increased in later sessions, which indicates that immersion increased over time and technology awareness was lessened. Immersion was more variable for Oculus Rift than Gear VR. Regarding VR dose, most 20-minute sessions were rated as too short. Ratings suggest that Veterans may prefer somewhat longer sessions for Gear VR than the more immersive Oculus Rift. Minimal adverse events, including cybersickness and the weight of the Gear VR aggravating pain, were reported. Finally, more than $30 \%$ of Veterans purchased VR HMDs or requested purchasing information during the study.

\section{Clinical Implications}

\section{Aim 1}

Considering the speed in which Veterans completed the hierarchy and their app intensity ratings, it is likely that the hierarchy was less incremental than designed. This would suggest that our approach to the hierarchy proved too conservative. Another possibility is that the intensity of their VR experience was shaped less by movement than their interest or engagement in the self-selected activities. The hierarchy will be modified before future use. The modification process will take a bottom-up approach to include Veteran stakeholders throughout this process of app selection, testing, and intensity rating [39]. Despite the movement heterogeneity in these Veteran-selected activities, these apps and their intensity ratings will be considered in the modification process. Having a structured app selection process for the hierarchy is important because of the goal to generalize to other VA hospitals and clinics. This is to ensure Veterans are not over- or underexposed to feared movements.

\section{$\operatorname{Aim} 2$}

Effect sizes for kinesiophobia and pain outcomes were smaller than in previous research. It is possible that VR is more efficacious for acute than chronic pain. However, these effects may have been attenuated by the ceiling and range restriction effects in the hierarchy. Moreover, given the wide CI and the small sample, estimation of CI at a lower limit of $75 \%$ to $85 \%$ may be appropriate to complement descriptive information in VR feasibility work [68]. When considering the MCID statistics in accordance with observed effect sizes for fear of movement (FDAQ), interference with mobility, pain intensity, pain catastrophizing, and patient-specific functioning may be promising for future research. Interestingly, Veteran-selected activities hindered by pain had the largest effect sizes and proportion of MCID. As discussed previously, Veteran-selected activities produced the highest VR intensity ratings. These findings highlight the importance of considering user preferences in selecting meaningful outcomes in addition to the VR intervention itself.

\section{$\operatorname{Aim} 3$}

Despite noted concerns with the hierarchy, this study further supports the feasibility of VR for pain management [30,69]. Specifically, the integration of VR technology itself was considered successful. Although VR was adjunct, Veterans both attended and completed sessions at high rates. Veterans typically rated VR sessions as too short, which was consistent with the success of a recent study that provided twelve 30-minute sessions using Oculus Rift [69]. It is notable that Veterans' ratings showed marginally lower preferences for 20-minute sessions using Oculus Rift. Given that immersion was also slightly higher for Oculus Rift than Gear VR, consideration for immersion level, HMD selection [70], and sensory demand [14,32] is important when considering session length with chronic pain populations.

\section{Limitations}

Consistent difficulties emerged when using Gear VR in this study. First, inconsistent hospital Wi-Fi hindered the importance of certain apps (eg, Guided Meditation). Second, Gear VR lacked a "kiosk mode" and apps would often time out and need to be restarted during sessions. This may have affected immersion. Third, Veterans indicated that environmental noise in the therapy gym was an issue, but only for Gear VR. This study used two Gear VR HMDs and one Oculus Rift. This approach was due to equipment availability and physical space considerations. The power supply on the gaming computer also shorted out during the first day of testing. Hence, only Gear VR was used during the first four testing days. These factors may have accelerated Veteran progress across the hierarchy. Use of a single HMD type-Oculus Rift—may be more beneficial for validation of VR hierarchies in busy medical settings.

Veterans used VR as an adjunct and were involved in on-going interdisciplinary pain management, which likely influenced Veteran improvement in treatment outcomes (see Murphy et al $[36,37])$. This may have also influenced Veterans' quick 
progressions across the hierarchy. Inclusion of a randomized control group is necessary to estimate the true added impact of VR versus treatment as usual.

This study had additional limitations, including a small sample size, which was limited by the allotted 3-week data collection period. This likely influenced the wide CI around the effect sizes [67]. Additionally, Veterans may not have been adequately challenged by the hierarchy. Finally, because this pilot study was unfunded, commercially available VR apps were used. A more optimal approach would be to develop or tailor VR apps to capture frequently avoided movements of varying intensity levels, such as safe strategies for bending or climbing stairs.

\section{Conclusions}

This study provided evidence that VR is feasible for chronic pain populations. In addition, this study expanded the knowledge base by demonstrating feasibility for VR as an adjunct for evidence-based chronic pain interventions in a medical setting. Implementation was more successful for VR technologies than the distraction-to-exposure hierarchy itself. Future research will focus on modification of this hierarchy to validate feared movements at varying intensity levels. This may be best accomplished using a bottom-up approach that includes Veterans in the intervention design and outcome selection processes.

\section{Acknowledgments}

The authors would like to thank Nicolle Angeli, PhD; Stacey Sandusky, PhD; and Megan Anderson, PTA, from the Chronic Pain Rehabilitation Program at the James A Haley Veterans Hospital for their assistance with participant recruitment, scheduling, and implementation strategies. Thank you to Spencer Mason from the Advanced Visualization Center at the University of South Florida for his assistance with VR technologies.

This material is the result of work supported with resources and the use of facilities at the James A Haley Veterans' Hospital. The contents of this manuscript do not reflect the views of the Department of Veterans Affairs of the United States Government.

\section{Conflicts of Interest}

JLM serves as the Chief Behavioral Health Officer of Karuna Labs. This is a recent appointment. JLM was not in this position during the conceptualization of this study, data collection, or drafting of this manuscript.

\section{References}

1. Dionisio JDN, Burns WGIII, Gilbert R. 3D Virtual worlds and the metaverse. ACM Comput Surv 2013 Jun 01;45(3):1-38. [doi: $10.1145 / 2480741.2480751]$

2. Parkin S. MIT Technology Review. 2014 Apr 23. Oculus Rift: thirty years after virtual-reality goggles and immersive virtual worlds made their debut, the technology finally seems poised for widespread use URL: https://www. technologyreview.com/s/526531/oculus-rift/ [accessed 2019-10-21]

3. Steuer J. Defining virtual reality: dimensions determining telepresence. J Comm 1992 Feb 07;42(4):73-93. [doi: 10.1111/j.1460-2466.1992.tb00812.x]

4. Garrett B, Taverner T, Masinde W, Gromala D, Shaw C, Negraeff M. A rapid evidence assessment of immersive virtual reality as an adjunct therapy in acute pain management in clinical practice. Clin J Pain 2014 Dec;30(12):1089-1098. [doi: 10.1097/AJP.0000000000000064] [Medline: 24535053]

5. Cummings JJ, Bailenson JN. How immersive is enough? A meta-analysis of the effect of immersive technology on user presence. Media Psychology 2015 May 15;19(2):272-309. [doi: 10.1080/15213269.2015.1015740]

6. Freeman D, Reeve S, Robinson A, Ehlers A, Clark D, Spanlang B, et al. Virtual reality in the assessment, understanding, and treatment of mental health disorders. Psychol Med 2017 Oct;47(14):2393-2400 [FREE Full text] [doi: 10.1017/S003329171700040X] [Medline: 28325167]

7. Olmos-Raya E, Ferreira-Cavalcanti J, Contero M, Castellanos MC, Giglioli IAC, Alcañiz M. Mobile virtual reality as an educational platform: a pilot study on the impact of immersion and positive emotion induction in the learning process. EURASIA J Math Sci Tech Ed 2018;14(6):2045-2057. [doi: 10.29333/ejmste/85874]

8. Trost Z, Parsons TD. Beyond distraction: virtual reality graded exposure therapy as treatment for pain-related fear and disability in chronic pain. J Appl Biobehav Res 2014 Jun 10;19(2):106-126. [doi: 10.1111/jabr.12021]

9. Chan E, Foster S, Sambell R, Leong P. Clinical efficacy of virtual reality for acute procedural pain management: a systematic review and meta-analysis. PLoS One 2018;13(7):e0200987 [FREE Full text] [doi: 10.1371/journal.pone.0200987] [Medline: 30052655]

10. International Association for the Study of Pain, Task Force on Taxonomy. Part III: a current list with definitions and notes on usage. In: Merskey H, Bogduk N, editors. Classification Of Chronic Pain: Descriptions Of Chronic Pain Syndromes And Definitions Of Pain Terms. Seattle, WA: IASP Press; 1994:207-214.

11. Treede R, Rief W, Barke A, Aziz Q, Bennett MI, Benoliel R, et al. A classification of chronic pain for ICD-11. Pain 2015 Jun;156(6):1003-1007 [FREE Full text] [doi: 10.1097/j.pain.0000000000000160] [Medline: 25844555]

12. Grichnik KP, Ferrante FM. The difference between acute and chronic pain. Mt Sinai J Med 1991 May;58(3):217-220. [Medline: 1875958] 
13. Nijs J, Paul van Wilgen C, Van Oosterwijck J, van Ittersum M, Meeus M. How to explain central sensitization to patients with 'unexplained' chronic musculoskeletal pain: practice guidelines. Man Ther 2011 Oct;16(5):413-418 [FREE Full text] [doi: 10.1016/j.math.2011.04.005] [Medline: 21632273]

14. Simons LE, Elman I, Borsook D. Psychological processing in chronic pain: a neural systems approach. Neurosci Biobehav Rev 2014 Feb;39:61-78 [FREE Full text] [doi: 10.1016/j.neubiorev.2013.12.006] [Medline: 24374383]

15. Murphy J, McKellar J, Raffa S, Clark M, Kerns R, Karlin B. Cognitive Behavioral Therapy for Chronic Pain Among Veterans: Therapist Manual. Washington, DC: US Department of Veterans Affairs; 2014.

16. Lethem J, Slade P, Troup J, Bentley G. Outline of a fear-avoidance model of exaggerated pain perception--I. Behav Res Ther 1983;21(4):401-408. [doi: $\underline{10.1016 / 0005-7967(83) 90009-8}$ ] [Medline: $\underline{6626110}$ ]

17. Vlaeyen JW, Linton SJ. Fear-avoidance and its consequences in chronic musculoskeletal pain: a state of the art. Pain 2000 Apr;85(3):317-332. [doi: 10.1016/s0304-3959(99)00242-0] [Medline: 10781906]

18. Leeuw M, Goossens ME, Linton SJ, Crombez G, Boersma K, Vlaeyen JW. The fear-avoidance model of musculoskeletal pain: current state of scientific evidence. J Behav Med 2007 Feb;30(1):77-94. [doi: 10.1007/s10865-006-9085-0] [Medline: 17180640]

19. Linton S, Shaw W. Impact of psychological factors in the experience of pain. Phys Ther 2011 May;91(5):700-711. [doi: 10.2522/ptj.20100330] [Medline: 21451097]

20. George SZ, Zeppieri G. Physical therapy utilization of graded exposure for patients with low back pain. J Orthop Sports Phys Ther 2009 Jul;39(7):496-505. [doi: 10.2519/jospt.2009.2983] [Medline: 19574658]

21. Smeets RJ, Vlaeyen JW, Hidding A, Kester AD, van der Heijden GJ, van Geel AC, et al. Active rehabilitation for chronic low back pain: cognitive-behavioral, physical, or both? First direct post-treatment results from a randomized controlled trial [ISRCTN22714229]. BMC Musculoskelet Disord 2006 Jan 20;7:5 [FREE Full text] [doi: 10.1186/1471-2474-7-5] [Medline: 16426449]

22. Parsons TD, Trost Z. Virtual reality-graded exposure therapy as treatment for pain-related disability in chronic pain. In: Ma M, Jain LC, Anderson P, editors. Virtual, Augmented Reality And Serious Games For Healthcare 1 (Intelligent Systems Reference Library). Berlin, Heidelberg, DE: Springer; Apr 26, 2014:523-546.

23. Hoffman HG, Chambers GT, Meyer WJ, Arceneaux LL, Russell WJ, Seibel EJ, et al. Virtual reality as an adjunctive non-pharmacologic analgesic for acute burn pain during medical procedures. Ann Behav Med 2011 Apr;41(2):183-191 [FREE Full text] [doi: 10.1007/s12160-010-9248-7] [Medline: 21264690]

24. McCaul KD, Malott JM. Distraction and coping with pain. Psychol Bull 1984 May;95(3):516-533. [Medline: 6399756]

25. Hoffman HG, Meyer WJ, Ramirez M, Roberts L, Seibel EJ, Atzori B, et al. Feasibility of articulated arm mounted Oculus Rift Virtual Reality goggles for adjunctive pain control during occupational therapy in pediatric burn patients. Cyberpsychol Behav Soc Netw 2014 Jun;17(6):397-401 [FREE Full text] [doi: 10.1089/cyber.2014.0058] [Medline: 24892204]

26. Malloy KM, Milling LS. The effectiveness of virtual reality distraction for pain reduction: a systematic review. Clin Psychol Rev 2010 Dec;30(8):1011-1018. [doi: 10.1016/j.cpr.2010.07.001] [Medline: 20691523]

27. Wiederhold BK, Gao K, Sulea C, Wiederhold MD. Virtual reality as a distraction technique in chronic pain patients. Cyberpsychol Behav Soc Netw 2014 Jun;17(6):346-352 [FREE Full text] [doi: 10.1089/cyber.2014.0207] [Medline: 24892196]

28. Jin W, Choo A, Gromala D, Shaw C, Squire P. A virtual reality game for chronic pain management: a randomized, controlled clinical study. Stud Health Technol Inform 2016;220:154-160. [Medline: 27046570]

29. Yilmaz Yelvar GD, Çırak Y, Dalkılınç M, Parlak Demir Y, Guner Z, Boydak A. Is physiotherapy integrated virtual walking effective on pain, function, and kinesiophobia in patients with non-specific low-back pain? Randomised controlled trial. Eur Spine J 2017 Feb;26(2):538-545. [doi: 10.1007/s00586-016-4892-7] [Medline: 27981455]

30. Thomas JS, France CR, Applegate ME, Leitkam ST, Walkowski S. Feasibility and safety of a virtual reality dodgeball intervention for chronic low back pain: a randomized clinical trial. J Pain 2016 Dec;17(12):1302-1317 [FREE Full text] [doi: 10.1016/j.jpain.2016.08.011] [Medline: 27616607]

31. Garcia-Palacios A, Botella C, Hoffman H, Fabregat S. Comparing acceptance and refusal rates of virtual reality exposure vs. in vivo exposure by patients with specific phobias. Cyberpsychol Behav 2007 Oct;10(5):722-724. [doi: 10.1089/cpb.2007.9962] [Medline: 17927544]

32. Parham LD, Cohn ES, Spitzer S, Koomar JA, Miller LJ, Burke JP, et al. Fidelity in sensory integration intervention research. Am J Occup Ther 2007;61(2):216-227. [doi: 10.5014/ajot.61.2.216] [Medline: 17436844]

33. Garrett B, Taverner T, Gromala D, Tao G, Cordingley E, Sun C. Virtual reality clinical research: promises and challenges. JMIR Serious Games 2018 Oct 17;6(4):e10839 [FREE Full text] [doi: 10.2196/10839] [Medline: 30333096]

34. US Department of Veterans Affairs. 2017. VA/DoD Clinical Practice Guideline for Opioid Therapy for Chronic Pain URL: https://www.healthquality.va.gov/guidelines/Pain/cot/ [accessed 2018-04-19]

35. Dahlhamer J, Lucas J, Zelaya C, Nahin R, Mackey S, DeBar L, et al. Prevalence of chronic pain and high-impact chronic pain among adults-United States, 2016. MMWR Morb Mortal Wkly Rep 2018 Sep 14;67(36):1001-1006 [FREE Full text] [doi: 10.15585/mmwr.mm6736a2] [Medline: 30212442]

36. Murphy JL, Clark ME, Banou E. Opioid cessation and multidimensional outcomes after interdisciplinary chronic pain treatment. Clin J Pain 2013 Feb;29(2):109-117. [doi: 10.1097/AJP.0b013e3182579935] [Medline: 22751033] 
37. Murphy JL, Phillips KM, Rafie S. Sex differences between Veterans participating in interdisciplinary chronic pain rehabilitation. J Rehabil Res Dev 2016;53(1):83-94 [FREE Full text] [doi: 10.1682/JRRD.2014.10.0250] [Medline: 27005932]

38. Krebs EE, Carey TS, Weinberger M. Accuracy of the pain numeric rating scale as a screening test in primary care. J Gen Intern Med 2007 Oct;22(10):1453-1458 [FREE Full text] [doi: 10.1007/s11606-007-0321-2] [Medline: 17668269]

39. Curran GM, Bauer M, Mittman B, Pyne JM, Stetler C. Effectiveness-implementation hybrid designs: combining elements of clinical effectiveness and implementation research to enhance public health impact. Med Care 2012 Mar;50(3):217-226 [FREE Full text] [doi: 10.1097/MLR.0b013e3182408812] [Medline: 22310560]

40. Birckhead B, Khalil C, Liu X, Conovitz S, Rizzo A, Danovitch I, et al. Recommendations for methodology of virtual reality clinical trials in health care by an international working group: iterative study. JMIR Ment Health 2019 Jan 31;6(1):e11973 [FREE Full text] [doi: 10.2196/11973] [Medline: 30702436]

41. Guided Meditation VR. 2016. URL: https://guidedmeditationvr.com [accessed 2018-06-11] [WebCite Cache ID 7067ZzUXl]

42. nDreams. 2016. Perfect URL: http://www.ndreams.com/titles/perfectvr/ [accessed 2018-06-11] [WebCite Cache ID 7068TH8zl]

43. Now VR. Rest VR: Relax and Meditate. 2016. URL: http://www.relaxvr.co/ [accessed 2018-06-11] [WebCite Cache ID 706I8RXQm]

44. Picselica. 2016. Ocean Rift URL: http://www.picselica.com/?cat=4 [accessed 2018-06-11] [WebCite Cache ID 706IYMIf8]

45. Greener Games. 2017. Nature Treks URL: http://greenergames.net [accessed 2018-06-11] [WebCite Cache ID 7069aNcDw]

46. Immersive Entertainment Inc. 2016. Grand Canyon VR URL: https://www.immersiveentertainment.com/ the-grand-canyon-vr-experience/ [accessed 2018-06-11] [WebCite Cache ID 706HP8KQ4]

47. Multiverse Entertainment. 2016. Reveries: Dream Flight URL: https://www.multiverseinc.com/dreamflight [accessed 2018-06-11] [WebCite Cache ID 706Ij5t5U]

48. Facebook. 2017. Paint VR URL: https://www.facebook.com/PAINTinVR/ [accessed 2011-06-11]

49. Google VR. 2017. Tilt Brush URL: https://www.tiltbrush.com [accessed 2018-06-11] [WebCite Cache ID 706HXsnr3]

50. Arrowiz. 2017. Beats Fever Paper URL: http://arrowiz.com/ [accessed 2018-06-11] [WebCite Cache ID 706IszBCR]

51. NiVision. The Show Must Go On. 2017. URL: https://nivisionag.wixsite.com/showmust-goon [accessed 2018-06-11] [WebCite Cache ID 706IEzvuJ]

52. Oculus VR. 2016. Oculus Rift URL: https://www.oculus.com/rift/ [accessed 2018-06-11] [WebCite Cache ID 706J17SJ1]

53. Samsung Oculus VR. 2015. Samsung Oculus Gear VR URL: https://www.oculus.com/gear-vr/ [accessed 2018-06-11] [WebCite Cache ID 706J6M3PU]

54. Czub M, Piskorz J. Body movement reduces pain intensity in virtual reality-based analgesia. Int J Hum-Comput Int 2017 Dec 08;34(11):1045-1051. [doi: 10.1080/10447318.2017.1412144]

55. Tashjian VC, Mosadeghi S, Howard AR, Lopez M, Dupuy T, Reid M, et al. Virtual reality for management of pain in hospitalized patients: results of a controlled trial. JMIR Ment Health 2017 Mar 29;4(1):e9 [FREE Full text] [doi: 10.2196/mental.7387] [Medline: 28356241]

56. Cole J, Crowle S, Austwick G, Slater DH. Exploratory findings with virtual reality for phantom limb pain; from stump motion to agency and analgesia. Disabil Rehabil 2009;31(10):846-854. [doi: 10.1080/09638280802355197] [Medline: 19191061]

57. Clark ME, Gironda RJ, Young RW. Development and validation of the Pain Outcomes Questionnaire-VA. J Rehabil Res Dev 2003;40(5):381-395 [FREE Full text] [Medline: 15080223]

58. George SZ, Valencia C, Zeppieri G, Robinson ME. Development of a self-report measure of fearful activities for patients with low back pain: the fear of daily activities questionnaire. Phys Ther 2009 Sep;89(9):969-979 [FREE Full text] [doi: 10.2522/ptj.20090032] [Medline: 19608630]

59. Salaffi F, Stancati A, Silvestri CA, Ciapetti A, Grassi W. Minimal clinically important changes in chronic musculoskeletal pain intensity measured on a numerical rating scale. Eur J Pain 2004 Aug;8(4):283-291. [doi: 10.1016/j.ejpain.2003.09.004] [Medline: 15207508$]$

60. Sullivan MJ, Bishop SR, Pivik J. The Pain Catastrophizing Scale: development and validation. Psychol Assessment 1995;7(4):524-532. [doi: 10.1037/1040-3590.7.4.524]

61. Osman A, Barrios FX, Kopper BA, Hauptmann W, Jones J, O'Neill E. Factor structure, reliability, and validity of the Pain Catastrophizing Scale. J Behav Med 1997 Dec;20(6):589-605. [Medline: 9429990]

62. Stratford P. Assessing disability and change on individual patients: a report of a patient specific measure. Physiother Can 1995 Oct;47(4):258-263. [doi: 10.3138/ptc.47.4.258]

63. Abbott JH, Schmitt J. Minimum important differences for the patient-specific functional scale, 4 region-specific outcome measures, and the numeric pain rating scale. J Orthop Sports Phys Ther 2014 Aug;44(8):560-564. [doi: 10.2519/jospt.2014.5248] [Medline: 24828475]

64. Kadel R, Kip K. A SAS macro to compute effect size (Cohen's d) its confidence interval from raw survey data. 2012 Oct 01 Presented at: SAS Global Forum 2012; April 22-25, 2012; Orlando, FL.

65. Cohen J. Statistical Power Analysis For The Behavioral Sciences (2nd Edition). New York: Routledge; 1988.

66. Tukey JW. Exploratory Data Analysis. Boston, MA: Pearson; 1977.

67. Cohen J. The earth is round (p<.05). Am Psychol 1994;49(12):997-1003. [doi: 10.1037//0003-066x.49.12.997] 
68. Lee EC, Whitehead AL, Jacques RM, Julious SA. The statistical interpretation of pilot trials: should significance thresholds be reconsidered? BMC Med Res Methodol 2014 Mar 20;14:41-49 [FREE Full text] [doi: 10.1186/1471-2288-14-41] [Medline: 24650044]

69. Garrett B, Taverner T, McDade P. Virtual reality as an adjunct home therapy in chronic pain management: an exploratory study. JMIR Med Inform 2017 May 11;5(2):e11 [FREE Full text] [doi: 10.2196/medinform.7271] [Medline: 28495661]

70. Tong X, Gromala D, Gupta D, Squire P. Usability comparisons of head-mounted vs. stereoscopic desktop displays in a virtual reality environment with pain patients. Stud Health Technol Inform 2016;220:424-431. [Medline: 27046617]

\author{
Abbreviations \\ CI: confidence interval \\ FDAQ: Fear of Daily Activities Questionnaire \\ HMD: head-mounted display \\ IQR: interquartile range \\ MCID: minimum clinically important difference \\ POQ-VA: Pain Outcomes Questionnaire-VA \\ PSFS: Patient-Specific Functional Scale \\ RCT: randomized controlled trial \\ VA: Veterans Affairs \\ VR: virtual reality
}

\author{
Edited by G Eysenbach; submitted 13.06.18; peer-reviewed by B Garrett, M Applegate; comments to author 10.07.18; revised version \\ received 13.09.19; accepted 26.09.19; published 30.10.19 \\ Please cite as: \\ Fowler CA, Ballistrea LM, Mazzone KE, Martin AM, Kaplan H, Kip KE, Ralston K, Murphy JL, Winkler SL \\ Virtual Reality as a Therapy Adjunct for Fear of Movement in Veterans With Chronic Pain: Single-Arm Feasibility Study \\ JMIR Form Res 2019;3(4):e11266 \\ URL: http://formative.jmir.org/2019/4/e11266/ \\ doi: $\underline{10.2196 / 11266}$ \\ PMID: 31670696
}

(C) Christopher A Fowler, Lisa M Ballistrea, Kerry E Mazzone, Aaron M Martin, Howard Kaplan, Kevin E Kip, Katherine Ralston, Jennifer L Murphy, Sandra L Winkler. Originally published in JMIR Formative Research (http://formative.jmir.org), 30.10.2019. This is an open-access article distributed under the terms of the Creative Commons Attribution License (https://creativecommons.org/licenses/by/4.0/), which permits unrestricted use, distribution, and reproduction in any medium, provided the original work, first published in JMIR Formative Research, is properly cited. The complete bibliographic information, a link to the original publication on http://formative.jmir.org, as well as this copyright and license information must be included. 